quintiles and for transformed z-scores (mean of 0 and SD of 1 ). Cox proportional hazards regression models were applied, stratified by cohort and corrected for measurement error.

Results In total 1312 primary brain tumours were diagnosed during follow-up, predominantly high-grade glioma $(n=436)$ and meningioma $(n=348)$. For meningioma, the HR was increased for systolic blood pressure $(\mathrm{HR}=1.27$ per unit $\mathrm{SD}, 95 \% \mathrm{CI} 1.03$ to 1.57$)$, for diastolic blood pressure ( $\mathrm{HR}=1.29,95 \% \mathrm{CI} 1.04$ to 1.58 ), and for the combined metabolic syndrome score $(\mathrm{HR}=1.31,95 \% \mathrm{CI} 1.11$ to 1.54). For high-grade glioma the risk was increased for diastolic blood pressure ( $\mathrm{HR}=1.23,95 \% \mathrm{CI} 1.01$ to 1.50$)$ and triglycerides $(\mathrm{HR}=1.35,95 \% \mathrm{CI} 1.05$ to 1.72$)$. For both meningioma and highgrade glioma, the risk was more than doubled in the fifth quintiles of diastolic blood pressure compared to the first quintile. For systolic blood pressure the meningioma risk was even larger.

Conclusion Increased blood pressure was related to risk of brain tumour, particularly of meningiomas.

\section{P2-70 PREVALENCE AND ASSOCIATED RISK FACTORS OF PERSISTENT ALBUMINURIA AMONG HYPERTENSIVE URBAN POPULATION OF KARACHI PAKISTAN}

doi:10.1136/jech.2011.142976i.5

M Ejaz, ${ }^{*}$ E Ahmed, J Hatcher, T Jafar. Aga Khan University Hospital, Karachi, Pakistan

Background Kidney failure is a worldwide public health problem, with increasing incidence and prevalence, high costs and poor outcome. Detection and treatment of chronic kidney disease, prior to kidney failure, is therefore of paramount importance. Albuminuria is one of the earlier markers of kidney damage and screening for albuminuria is recommended in high risk patients.

Objective To determine the prevalence and associated risk factors of persistent albuminuria, defined as urine albumin concentration of $20 \mathrm{mg} / \mathrm{l}$ and urine sex-specific albumin-to-creatinine ratio of 20 and $30 \mathrm{mg} / \mathrm{g}$ for males and females respectively persisting for more than 3 months in hypertensive population.

Methods $72 \%$ of 1340 newly diagnosed hypertensive subjects from ongoing community based cohort study who had been screened once for the presence of albuminuria $(n=240)$, were retested for the presence of persistent albuminuria in this study. Albumin concentration $(\mathrm{mg} / \mathrm{l})$ and albumin-to-creatinine ratio $(\mathrm{mg} / \mathrm{gm})$ were determined in a spot morning urine sample by Nephlometry.

Results Prevalence of persistent abnormal albuminuria 9.3\% [7.8\%$10.8 \%$. Mean age $52( \pm 11.4)$ years and $58 \%$ were males. Factors independently associated were male gender (OR 2.0 (1.24-2.97)), young age with positive family history of $\mathrm{KD}$ disease (OR 15.51 (7.35-32.97)). Off the measurable variables high cholesterol levels $(p=<0.001)$, and progressively higher levels of systolic BP $(p<0.001)$ were associated risk factors.

Conclusion Hypertensive kidney damage is already present in a significant number among newly diagnosed hypertensive population suggesting late detection of high blood pressure. Public awareness through education is necessary in developing countries to detect hypertension before target organ damage has occurred.

\section{P2-71 SOCIOECONOMIC INEQUALITIES IN OVERWEIGHT AMONG ADULTS IN TURKEY: A REGIONAL EVALUATION}

doi:10.1136/jech.2011.142976i.6

${ }^{1} \mathrm{I}$ Ergin, ${ }^{*}{ }^{1} \mathrm{H}$ Hassoy, ${ }^{2} \mathrm{~A}$ Kunst. ${ }^{1}$ Ege University Department of Public Health, Izmir, Turkey; ${ }^{2}$ Academic Medical Centre (AMC), University of Amsterdam, Department of Public Health, Amsterdam, The Netherlands

Introduction Patterns of socioeconomic inequalities in obesity and overweight have not been documented for Turkey. This study aimed to describe educational and wealth-related inequalities for overweight in Turkey, taking a regional perspective.

Methods Data from the World Health Survey 2002 for Turkey was used. Among the respondents 20 years and older, 3790 women and 4057 men had data on self-reported height and weight. Respondents were classified according to education years and a wealth score derived from the availability of household assets. Logistic regression analysis was applied to assess the relationship between overweight and socioeconomic factors. Analyses were stratified by sex and region (West, Mediterranean, Middle, Black Sea, East).

Results Age-adjusted overweight prevalence is $48.4 \%$ for women and $46.1 \%$ for men. For men, education was not systematically related to overweight while overweight was significantly increased among the highest wealth groups. For women, the prevalence of overweight was highest for low-educated and middle-wealth groups. The size of the inequalities in overweight showed only small regional variations. In the East, however, overweight prevalence was more related to higher socioeconomic position than in the other regions.

Conclusion Socioeconomic inequalities for overweight in Turkey are at a similar level as in most European countries, and especially comparable to southern Europe. The smaller inequalities in the East correspond to the low level of socioeconomic development at this part of the country.

\section{P2-72 IMPACT OF THE INTRODUCTION OF SELECTIVE COX-2 INHIBITORS ON HOSPITALISATION FOR GASTRIC BLEEDING IN AUSTRALIA}

doi:10.1136/jech.2011.142976i.7

${ }^{1}$ A Esterman, ${ }^{*} \mathrm{G}$ Young. ${ }^{1}$ University of South Australia, Adelaide, South Australia, Australia; ${ }^{2}$ Flinders University, Adelaide, South Australia, Australia

Background Selective COX-2 inhibitors (coxibs) have been shown to be advantageous for gastrointestinal safety in several large studies but whether this translates into fewer events at the population level is unclear.

Aim To examine the relationship between prescriptions for nonselective NSAIDs, selective COX-2 inhibitors (coxibs), H2-receptor antagonists (H2RA) and protein pump inhibitors (PPI), and hospital separations for upper gastro-intestinal haemorrhage (UGH) in Australia, over the period 1998-2002.

Methods Interrupted time series study. Monthly hospital separations for UGH for Australia for the period July 1998 to June 2002 were obtained from the Australian Institute for Health and Welfare. Data on prescriptions for coxibs and non-selective NSAIDs, PPIs and H2RAs were obtained from commercial sources for Australia for the same period. Rates of UGH per 1000 prescriptions for all NSAIDs were modelled by Poisson regression. The period before the introduction of coxibs (1998-1999) was compared with the period after their introduction (20012002).

Results Rates for UGH per 1000 prescriptions for all NSAIDs significantly declined between the two periods. Adjusting for prescriptions for PPIs and H2RAs improved the fit of the model and after adjustment, the reduction in UGH separation rates per 1000 prescriptions for all NSAIDs was estimated to be $9.5 \%$ (95\% CI $9.1 \%$ to $10.9 \%$ ).

In this presentation, we will also provide extended results covering the period January 1997 to October 2010, and also including sales of anti-ulcerants and other non-steroidal antirheumatics in the model. 